\title{
Antiangiogenic activity of iridoids from Lamiaceae and Plantaginaceae species
}

Anna Maria Iannuzzi ${ }^{1}$, César Muñoz Camero ${ }^{1}$, Khadidja Aya Beladjila ${ }^{2}$, Marinella De Leo ${ }^{1}$, Valeria D’Angelo ${ }^{3}$, Maria Paola Germanò ${ }^{3}$, Smain Amira ${ }^{4}$, Ammar Bader $^{5}$, Nunziatina De Tommasi ${ }^{6}$, Alessandra Braca ${ }^{1}$

${ }^{1}$ Dipartimento di Farmacia, Università di Pisa, Pisa, Italy

2 Laboratoire d'Obtention des Substances Thérapeutiques (LOST), Département de Chimie, Université des Frères Mentouri-Constantine, Constantine, Algeria

${ }^{3}$ Dipartimento di Scienze Chimiche, Biologiche, Farmaceutiche e Ambientali, Università degli Studi di Messina, Messina, Italy

${ }^{4}$ Department of Animal Biology and Physiology, University of Setif, Setif, Algeria

${ }^{5}$ Department of Pharmacognosy, Faculty of Pharmacy, Umm Al-Qura University, Makkah, Saudi Arabia

${ }^{6}$ Dipartimento di Farmacia, Università di Salerno, Fisciano (SA), Italy

Iridoids are a group of natural compounds, occurring in a great number of plant families, usually as glycosides. The considerable interest in iridoids is due to their ecological role as plant protectant and to their wide spectrum of biological activities, including cardioprotection, neuroprotection, antiinflammatory, and anticancer activities [1]. Interestingly, some iridoid glycosides were found to have a potent antiangiogenic activity [2-3]. Angiogenesis process may be involved in tumour development, thus its inhibition appears to be a promising approach in anti-inflammatory and anticancer therapies [4]. Within this context, the aim of the present study was the isolation and characterization of iridoid derivatives from two Lamiaceae species, Stachys ocymastrum (L.) Briq and Premna resinosa (Hochst.) Schauer leaves, and from Anarrhinum pedatum Desf. aerial parts, belonging to Plantaginaceae family, together with the evaluation of their antiangiogenic potential. The chemical study of investigated plants afforded to the isolation of one new and four known iridoid glycosides from $S$. ocymastrum, nine known iridoid diglycosides from $P$. resinosa, and ten new and five known iridoid glycosides from $A$. pedatum, identified by NMR and MS analyses. The antiangiogenic effects of the isolates were reported on new blood vessels formation using two in vivo models: zebrafish embryos and chick embryo chorioallantoic membrane [5]. Among the tested iridoids, $\beta$ hydroxyipolamiide, ipolamiide, buddlejoside $\mathrm{A}_{5}$, and 6'-O-menthiafoloylmussaenosidic acid-11-(5$O$ - $\beta$-D-fructopyranosyl) ester showed a significant antiangiogenic activity in both assays, reducing the growth of blood vessels. Weaker antiangiogenic effects were also observed for some other iridoids, thus suggesting this class of compounds as promising antiangiogenic agents. 


\section{References:}

[1] Leticia J, Jack, LB. Iridoids. A review. J Nat Prod 1980; 43: 649-707.

[2] Munoz Camero C, Germanò MP, Rapisarda A, D’Angelo V, Amira S, Benchikh F, Braca A, De Leo M. Anti-angiogenic activity of iridoids from Galium tunetanum. Braz J Pharmacog 2018; 28: 374-377.

[3] Koo H-J, Lee S, Shin K-H, Kim B-C, Lim C-J, Park E-H. Geniposide, an anti-angiogenic compound from the fruits of Gardenia jasminoides. Planta Med 2004; 70: 467-469.

[4] Folkman J. Angiogenesis in cancer, vascular, rheumatoid and other disease. Nat Med 1995; 1, 27 31.

[5] Certo G, Costa R, D’Angelo V, Russo M, Albergamo A, Dugo G, Germanò MP. Anti-angiogenic activity and phytochemical screening of fruit fractions from Vitex agnus castus. Nat Prod Res 2017; 31: 2850-2856.

Preferred presentation: poster 